\title{
Neue Herausforderungen für biometrische Technologien
}

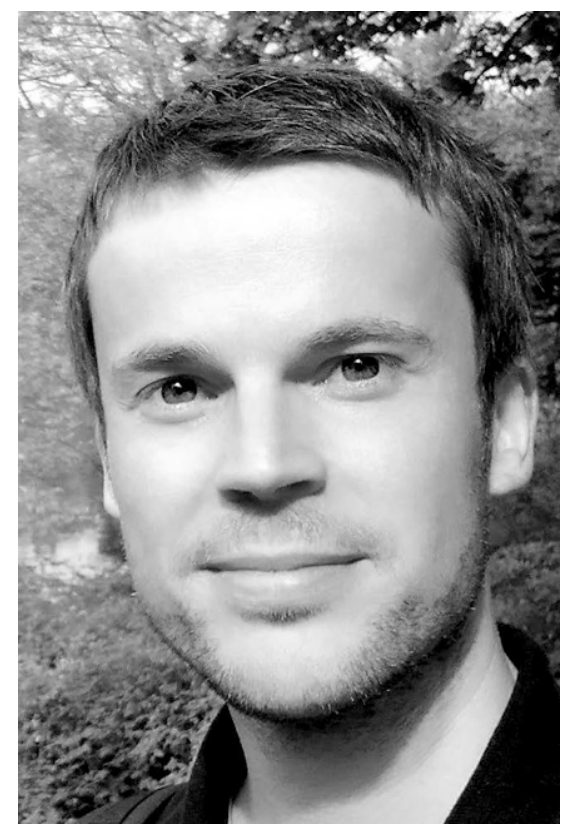

Biometrische Technologien sind ein integraler Bestandteil in modernen Identitätsmanagementsystemen geworden. Im Gegensatz zu Besitz- oder Wissensbasierten Authentifikationssystemen können Biometrische Charakteristika, z.B. Fingerabdruck oder Gesicht, nicht verloren oder an Dritte weitergegeben werden. Diese Vorteile verbessern sowohl die Benutzbarkeit als auch die Sicherheit von Authentifikationssystemen. Der stetig wachsende Einsatz von biometrischen Systemen führt zu neuen operativen, sicherheitsrelevanten sowie datenschutzrechtlichen Herausforderungen, die technologisch gemeistert werden müssen.

Aufgrund der Tatsache, dass viele etablierte biometrische Charakteritika nicht geheim sind, insbesondere das Gesicht oder Fingerabdrücke, sind sogenannte Präsentationsattacken oder auch "Spoofing"-Attacken höchst sicherheitskritisch. In jüngster Vergangenheit zeigten Forscher zusätzlich, dass biometrische Systeme auch verwundbar gegenüber sogenannten "Morphing-Angriffen" sind. Diese Herausforderungen werden in diesem Heft behandelt: Gomez-Barrero et al. diskutieren den aktuellen Stand der Technik sowie Herausforderungen hinsichtlich der Erkennung von Präsentationsangriffen auf Fingerabdruckverfahren. Merkle et al. geben einen Einblick in Morphing-Angriffe auf Gesichtserkennungssysteme, und schaffen einen Überblick über den derzeitigen Stand der Technik bzgl. Methoden zur Detektion von gemorphten Gesichtsbildern.

Der flächendeckende (trans-)nationale Einsatz biometrischer Technologien, etwa zum Zweck der Überwachung von Grenzübertritten in und aus dem Schengen-Raum im geplanten EU Einreise-/Ausreisesystem, birgt weitere Herausforderungen. Während die Interoperabilität durch die Erfüllung biometrischer Standards gewährleistet sein muss, sollten die sich ständig weiterentwickelnden biometrischen Technologien objektiv mittels standardisierter Methoden bewertet werden. Des Weiteren muss eine entsprechende Qualität biometrischer Daten sichergestellt werden, um eine hinreichende Erkennungsgenauigkeit zu garantieren. Eine Einführung zu neuen Normen für biometrische Datenaustauschformate wird von Henniger gegeben. Von Twickel et al. diskutieren die Vereinbarkeit der Erhebung und Verarbeitung biometrischer Daten zum Zweck der Evaluation biometrischer Systeme. Tams und Schwaiger heben die Wichtigkeit biometrischer Qualitätsanforderungen durch eine Diskussion der möglichen Auswirkung verschiedener Varianten zur Erfassung von Gesichtsbildern an den Grenzkontrollstationen hervor.

Genannte Herausforderungen können teilweise durch den Einsatz neuer biometrischer Modalitäten sowie einer Verknüpfung neuartiger Technologien mit biometrischen Systemen gelöst werden. Uhl gibt einen Überblick über den derzeitigen Stand der Technik von Venenerkennungssystemen. Abschließend diskutieren Buchmann und Margraf die Möglichkeit langfristige Sicherheit für sogenannte BreederDokumente durch den kombinierten Einsatz von Biometrie und Blockchain-Technologie zu gewährleisten.

Während biometrische Technologien bereits täglich Anwendung finden, etwa bei der automatischen Grenzkontrolle an Flughäfen oder zum Entsperren von Smartphones, zeigen die Beiträge, dass viele neue Herausforderungen noch gelöst werden müssen. Diese Beobachtung unterstreicht die Bedeutung und Notwendigkeit der Forschung im Bereich Biometrie.

\section{Christian Rathgeb}

